\title{
空间调制全偏振计算成像场景迁移超分辨率方法
}

\author{
徐国明 ${ }^{1,2,3)}$, 袁宏武 ${ }^{2,3)^{*}}$, 薛模根 ${ }^{3)}$, 王峰 ${ }^{3)}$, 王杰 ${ }^{1)}$ \\ 1) (安徽大学互联网学院 合肥 230039) \\ ${ }^{2)}$ (安徽新华学院大数据与人工智能学院 合肥 230088) \\ 3) (陆军炮兵防空兵学院偏振光成像探测技术安徽省重点实验室 合肥 230031) \\ (89662550@qq.com)
}

\begin{abstract}
摘 要: 空间调制型全偏振计算成像同时获得可见光和红外通道图像, 但是受探测器限制, 2 个通道的图像空间分辨 率不一致, 给后端的图像融合及目标探测过程带来不便. 为此, 提出基于场景特征迁移学习的空间调制型计算成像 超分辨率方法. 首先在分析空间调制计算成像的相同场景异源图像特点基础上，构建场景特征迁移模型; 然后建立 改进场景迁移卷积神经网络结构并选择修正线性激活函数，同时增加空间分辨率一致性约束; 再设计最优光谱迁移 响应学习策略, 并作为前端输人加到超分辨率网络; 最后将光谱迁移响应优化与全偏振超分辨率重建的参数联合学 习, 获得高分辨率偏振图像. 利用实际成像系统的仿真数据及系统数据进行 2 倍和 3 倍超分辨率实验, 从主观视觉效 果、客观量化评价指标和偏振参量解析结果 3 个方面对多个指标进行评价. 结果表明, 文中方法在视觉效果上能够保 持目标轮廓并抑制噪声干扰，在 16 个客观指标对比数据上取得了 10 个优于、 3 个相同和 3 个低于的结果, 验证了该 方法的有效性, 为成像系统定标校正提供数据支持.
\end{abstract}

关键词: 全偏振; 超分辨率; 计算成像; 迁移学习

中图法分类号: TN911.73 DOI: 10.3724/SP.J.1089.2021.18699

\section{Spatial Modulation Full Polarization Computing Imaging Super-Resolution via Scene Transfer}

\author{
Xu Guoming ${ }^{1,2,3)}$, Yuan Hongwu ${ }^{2,3)^{*}}$, Xue Mogen ${ }^{3)}$, Wang Feng ${ }^{3)}$, and Wang Jie ${ }^{1)}$ \\ 1) (School of Internet, Anhui University, Hefei 230039) \\ 2) (School of Big Data and Artificial Intelligence, Anhui Xinhua University, Hefei 230088) \\ 3) (Anhui Province Key Laboratory of Polarized Imaging Detecting Technology, Army Artillery and Air Defense Forces Academy of PLA, Hefei \\ 230031)
}

\begin{abstract}
The spatial modulation full polarization computing imaging can obtain both visible and infrared channel images synchronously. However, due to the limitation of the detector, the spatial resolution of the two channels is inconsistent, which brings inconvenience to the following image fusion and target detection process. A spatial modulation computing imaging super-resolution method via scene feature transfer learning is proposed. Firstly, the scene feature transfer model is constructed. The model is based on the analysis of the spatial modulation computing imaging characteristics of different source images in the same scene. Secondly, the convolutional neural networks $(\mathrm{CNN})$ structure of scene transfer is improved and the rectified linear unit
\end{abstract}

收稿日期：2020-10-07; 修回日期：2021-04-30. 基金项目：国家自然科学基金(61379105); 安徽省自然科学基金(2108085MF230, 1908085MF208); 中国博士后科学基金(2016M592961); 陆军装备部十三五预研子课题; 安徽省高校自然科学研究重点项目 (KJ2018A0587, KJ2019A0906). 徐国明(1979-), 男, 博士, 副教授, 硕士生导师, CCF 高级会员, 主要研究方向为偏振成像探测、图像 表示与超分辨率; 袁宏武(1979-), 男, 博士, 副教授, 论文通讯作者, 主要研究方向为图像融合、深度学习; 薛模根(1964一), 男, 博 士, 教授, 博士生导师, 主要研究方向为光电防御; 王峰(1972-), 男, 博士, 教授, 主要研究方向为偏振成像技术; 王杰(1996一), 男, 硕士研究生, 主要研究方向为图像超分辨率. 
activation function is selected. At the same time, the spatial resolution consistency constraint is added. Then, the optimal spectral transfer response learning strategy is designed and added to the super-resolution network as the front-end input. Finally, the parameters of spectral transfer response optimization and full polarization super-resolution reconstruction are learned together to obtain the high-resolution polarization image. Some super-resolution experiments with scale factors 2 and 3 are carried out using simulation data and system data of the actual imaging system. The experiments result is evaluated by multiple indicators with three aspects such as subjective visual effect, objective quantitative index and polarization parameter analysis results. In the visual effect, the method can keep the object contour and suppress the noise interference. On the 16 objective index data, the method obtains 10 better, 3 equal and 3 lower results compared with the others. The results verify the effectiveness of the method and also provide data support for calibration correction of imaging system.

Key words: full polarization; super resolution; computing imaging; transfer learning

空间调制型全偏振成像技术是继传统的分时 和同时偏振成像技术后发展起来的新体制偏振成 像技术，在新的成像体制下，系统利用 Savart 偏光 镜将被探测目标的 4 个 Stokes 矢量调制在同一幅干 涉图像中，从而通过单次采集便可获得完整的偏 振信息 ${ }^{[1-2]}$. 该体制以其同时获得多个 Stokes 矢量、 结构简单易于实现以及可对动态目标成像等优点 逐渐成为研究热点 ${ }^{[3]}$. 传统成像方法通过在传感器 像元与场景之间建立一种直接的映射函数获取图 像，而新的计算成像体制 ${ }^{[4]}$ 将成像系统与后置的特 征提取和图像重建看作一个整体，通过对所获取 的干涉图采样电荷耦合器件(charge coupled device, CCD)进行离散傅里叶变换, 便可以解调出 目标的辐射光谱和偏振信息。该体制能够利用高 性能的计算能力及全局化的信息处理能力，在超 衍射极限成像、大视场高分辨率(high resolution, HR)成像及透过散射介质清晰成像等应用中发挥 作用.

在实际应用中，成像系统的分辨率对目标探 测和识别精度影响重大. 由于光学器件决定了成 像系统的图像最高空间分辨率，因此在目标识别 和定量分析等实际应用时，通常采用超分辨率 (super resolution, SR)方法获取高于成像系统分辨率 的图像. 例如, 2018 年 5 月，我国发射的高分 5 号 卫星(GF-5)所搭载的多角度偏振成像仪采用面阵 $\mathrm{CCD}$ 探测器 ${ }^{[5]}$, 图像分辨率为 $512 \times 512$ 像素, 星下 点像元大小为 $3.3 \mathrm{~km}$. 通过融合高分辨率的全色影 像和低分辨率(low resolution, LR)的高光谱影像，可 重建得到同时具有高空间分辨率和高光谱分辨率 的遥感影像，从而达到提升性能、扩展应用范围的 目的 ${ }^{[6]}$. 同时，基于深度学习和样本特征融合的高
光谱图像 SR 研究近年来受到了广泛关注 ${ }^{[7-9]}$, 这些 方法通过对成像降质过程建模，采取监督或半监 督学习，在提高光谱分辨率的同时能有效地改善 图像空间分辨率. 但是，此类方法用于空间调制型 计算成像时存在 SR 方法在真实场景上应用的普遍 问题 ${ }^{[10]}$ : 一是依赖良好的训练样本, 缺少高-低分 辨率图像对，而这样的样本在新体制成像技术和 特殊应用领域中不易获取; 二是采用端到端的学 习容易忽略计算成像的图像质量退化与重建过程. 对于样本依赖问题，近年来基于小样本元学习的 $S R$ 方法开始受到关注 ${ }^{[11-12]}$, 文献[13-14]则从图像 退化模型中学习 SR 参数以提高重建效果.

对于空间调制型计算成像，其图像退化过程 不仅包括传统成像方法在传感器像元与场景之间 的直接映射模型，该映射关系对应空间调制型全 偏振计算成像的 CCD 上的干涉条纹强度图像; 还 包括利用二维离散傅里叶变换将空间域干涉条纹 信息转换到频域的计算成像，采用低通滤波器计 算获得目标的 Stokes 矢量信息的空间调制过程 ${ }^{[2]}$. 同时，在具体高光谱全偏振成像系统中，除了获取 探测目标的偏振信息外，还获得目标的高光谱信 息及高分辨率的可见光全色图像，这些异源的圥 余高光谱和可见光图像为相同目标场景的低分辨 率偏振图像 SR 方法提供了额外的目标场景先验. 近年来, 迁移学习方法 ${ }^{[12,15-16]}$ 为利用场景先验进行 $\mathrm{SR}$ 提供了思路和技术手段，代表性的方法是利用 HR RGB 图像先验信息提升高光谱图像 $\mathrm{SR}^{[17-19]}$. 实 际成像探测中, 高光谱成像系统为了达到高光谱 分辨率往往会牺牲时间和空间分辨率，而可见光 (或多光谱)相机集成了宽波长范围的辐射，可以 轻松地实时捕捉高空间分辨率的图像。受此启发, 
本文针对空间调制型计算成像降质过程及成像系 统特点, 融合相同目标场景 HR 的异源图像与 HR 可见光图像之间的空间一致性，在卷积神经网络 (convolutional neural networks, CNN)框架下, 提出 基于场景特征迁移的空间调制型计算成像 SR 方法.

\section{1 空间调制型高光谱全偏振系统}

\section{1 成像系统}

空间调制型全偏振成像是继传统的分时和同 时偏振成像后发展起来的新型偏振成像体制. 图 1 所示为某型 2 通道高光谱全偏振同时成像仪. 系统 主要由前置扩束光学器件 (beam expander optics, $\mathrm{BEO})$ 、半波片前表面孔径光栏(S)、Savart 偏光镜 (Savart polariscope, SP)、液晶调谐滤光片 $(\mathrm{P}) 、$ 计算 成像系统(computing imaging system, CIS)和 CCD 构成. 其中, 面阵探测器的可见光近红外波段分辨 率为 $2048 \times 2048$, 像元尺寸为 $12 \mu \mathrm{m}$; 短波红外波 段分辨率为 $640 \times 512$, 像元尺寸为 $20 \mu \mathrm{m}$.

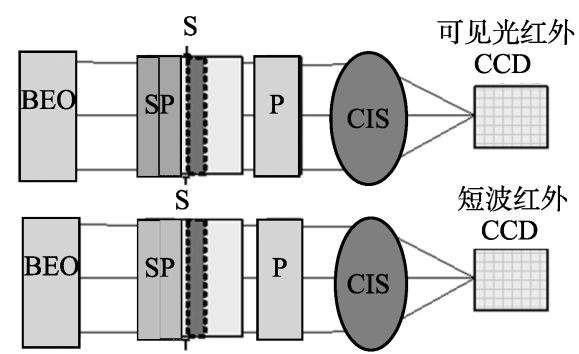

图 1 全偏振成像仪的基本组成

该成像系统采用 Stokes 矢量的空间调制原理, 同时将 4 个 Stokes 矢量 $\left(\boldsymbol{S}_{0} \sim \boldsymbol{S}_{3}\right)$ 调制在同一幅图像 中, 一次采集即可得到包含目标 4 个 Stokes 矢量的 调制信息, 据此可以解析出多个偏振参量图像 ( $\boldsymbol{I}, \boldsymbol{Q}, \boldsymbol{U}$ 和 $\boldsymbol{V}$ 等)。高光谱的实现通过液晶调谐滤 光片快速切换波段, 实现目标完整偏振状态的快 速测量, 从不同角度反映出场景和目标信息, 实现 目标全偏振参量同步探测.

\section{2 偏振解调计算成像}

空间调制器模块由一对 SP、半波片 (half-wave plate, HWP)和一块偏振片 (analyzer)组成, 如图 2 所 示. 人射光束经过第 1 个 SP 后分成 2 束光，经过与 $X$ 轴夹角为 $22.5^{\circ}$ 的 HWP 和第 2 个 SP(与前一 SP 正交)后，光束分成 4 条; 再经过与 $X$ 轴夹角 $45^{\circ}$ 的 偏振器和成像物镜, 4 条光束在探测器面上相遇, 满足干涉条件, 在探测器面上形成网格状的干涉 条纹强度图像 ${ }^{[20]}$.

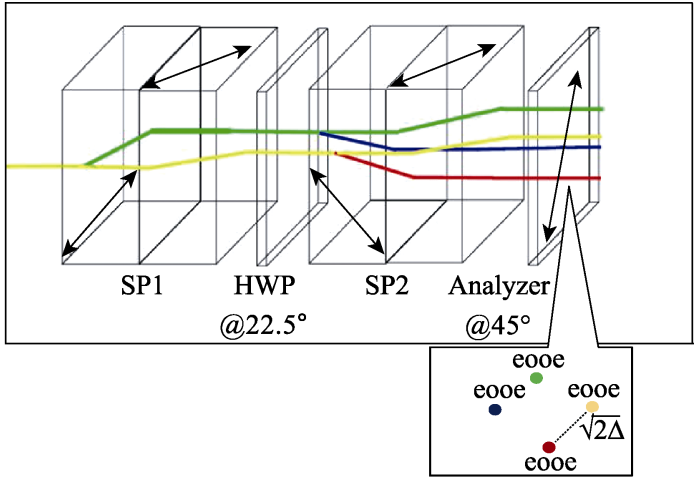

图 2 基于 SP 的空间调制模块

SP 对每物点光束在空间上先分解调制再合成, 使每像点光强为 4 个 Stokes 矢量的线性叠加. 对于 空间调制型计算成像，其偏振参量图像首先通过 波动光学理论获得 $\mathrm{CCD}$ 上的干涉强度图像与 Stokes 偏振参量的关系; 然后在频域进行解调，用 二维离散傅里叶变换将空间域干涉条纹信息转换 到频域, 采用低通滤波器解算获得目标的 Stokes 矢 量信息, 解调方法为

$$
\left\{\begin{array}{l}
F\left[\boldsymbol { S } _ { 0 } ( x , y ) \left[=F_{0}(u, v) \leftrightarrow \boldsymbol{S}_{0}(x, y)=F^{-1}\left[F_{0}(u, v)\right]\right.\right. \\
F\left\{\boldsymbol{S}_{1}(x, y) \exp [\mathrm{i} 2 \pi \Omega(x+y)]\right\}=F_{1}(u-\alpha, v-\alpha) \leftrightarrow \\
\boldsymbol{S}_{1}(x, y)=F^{-1}\left[F_{1}(u-\alpha, v-\alpha)\right] \exp [-\mathrm{i} 2 \pi \Omega(x+y)] \\
F\left[\boldsymbol{S}_{23}(x, y) \exp (-\mathrm{i} 4 \pi \Omega x)\right]=F_{23}(u+2 \alpha, v) \leftrightarrow \\
\boldsymbol{S}_{23}(x, y)=F^{-1}\left[F_{23}(u+2 \alpha, v)\right] \exp (\mathrm{i} 4 \pi \Omega x) \\
\boldsymbol{S}_{2}(x, y)=\operatorname{Re}\left[\boldsymbol{S}_{23}(x, y)\right] \\
\boldsymbol{S}_{3}(x, y)=\operatorname{Im}\left[\boldsymbol{S}_{23}(x, y)\right]
\end{array}\right.
$$

其中, $\Omega$ 为载波频率; $x, y$ 为像平面坐标; 位移量 $a=\Omega \cdot S \cdot K ; S$ 为 $\mathrm{CCD}$ 单个像素尺寸; $K$ 为 $\mathrm{CCD}$ 行(列)包含的像素数(默认行列像素数相等).

该解调计算成像过程中, 如果频域滤波器带 宽超过一定范围 $a$, 该分量的频谱会被其他分量频 谱覆盖, 重构图像将出现明显的混叠. 因此必须限 制滤波器的带宽，而带宽限制必然造成图像高频 信息的损失，图像分辨率降低。一方面，要合理 设置系统参数, 提升图像重构效果; 另一方面, 在主控端的显控和输出中, 需要根据实际应用进 行 SR 重建.

\section{3 异源图像特征}

在全偏振成像系统中，视频图像采集一共有 2 路：一路是可见光近红外(空间分辨率为 $2048 \times$ 2048 , 图像格式为 12 位); 另一路是短波红外(空间 分辨率为 $640 \times 512$, 图像格式为 14 位), 帧频都是 20 帧/s. 传输至计算机中的图像数据为探测器采集得 到的 2 路干涉图像, 再分别经由偏振解调算法解析 出 4 个 Stokes 矢量. 
2 路图像输出和处理方式如下: (1) 偏振成像 模式, 实现多种偏振参量图像的独立成像或多参 量融合成像; (2) 光谱成像模式, 实现波长为 $420 \sim 1700 \mathrm{~nm} ， 64$ 个波段的光谱成像；(3) 可见/近 红外光强融合成像模式，实现 2 个波段的光强融合 成像; (4) 偏振/光谱融合成像模式, 实现上述 3 种 模式的融合成像.

在实际成像探测应用中，通常，为了综合利用 多源图像特征以提高探测和识别精度, 需要对图 像进行融合处理，而在不同空间分辨率的状态下 作像素级融合实现困难，于是基于信号处理的 SR 技术被广泛应用. 图 1 中, 由于可见光近红外和短 波红外经光学校正和配准后是对同一场景目标进 行成像, 因此 HR 的可见光偏振图像就为 LR 红外 偏振图像 SR 提供场景先验信息, 同时, 相同成像 条件的噪声也为迁移学习提供损失先验.

\section{2 场景特征迁移模型}

\section{1 问题描述}

在第 1.1 节的成像系统中, 用 $\boldsymbol{X}_{\mathrm{L}} \in \mathbb{R}^{m \times n}$ 表示 $\mathrm{LR}$ 红外偏振图像, HR 可见光偏振图像描述为 $\boldsymbol{Y} \in \mathbb{R}^{M \times N}$, 通过场景光谱迁移响应进行融合, 得 到 $\mathrm{HR}$ 红外偏振图像 $\boldsymbol{X} \in \mathbb{R}^{M \times N}$; 其中, $M$ 和 $N$ 为 重建的 HR 红外偏振图像 $\boldsymbol{X}$ 的行数和列数, 相应 的, $m$ 和 $n$ 为 LR 图像 $\boldsymbol{X}_{\mathrm{L}}$ 的行数和列数. 输人的低 分辨率图像 $\boldsymbol{X}_{\mathrm{U}}$ 是 $\boldsymbol{X}$ 在像元合并空间域的下采样 输出, HR 可见光偏振 $\boldsymbol{Y}$ 可以通过场景偏振光谱迁 移下采样 $\boldsymbol{X}$ 得到. 在高光谱全偏振混合成像系统 中, 经系统定标和光学校正后, $\boldsymbol{X}_{\mathrm{U}}$ 和 $\boldsymbol{Y}$ 之间的场 景误差可以忽略, 则 LR 红外偏振图像 $\boldsymbol{X}_{\mathrm{L}}, \mathrm{HR}$ 的 红外偏振图像 $\boldsymbol{X}$ 和可见光偏振图像 $\boldsymbol{Y}$ 之间的关系 可以用线性方法来描述, 表示为

$$
\boldsymbol{X}_{\mathrm{L}}=\boldsymbol{X} H
$$

$$
\boldsymbol{Y}=C \boldsymbol{X}
$$

其中, $H \in \mathbb{R}^{M N \times m n}$ 为空间调制过程中的像元合并下 采样卷积操作; $C \in \mathbb{R}^{b \times B}$ 为将偏振光谱从红外通道 转换到可见光通道的场景偏振光谱迁移响应函数, $b$ 和 $B$ 分别表示可见光和红外通道的光谱波段. 由此, 空间调制型红外偏振图像 SR 可以建模为 LR 红外偏 振图像和 HR 可见光偏振图像的融合过程, 即

$$
E(\boldsymbol{X})=E_{\mathrm{d}}\left(\boldsymbol{X}, \boldsymbol{X}_{\mathrm{L}}, \boldsymbol{Y}\right)+\lambda E_{\mathrm{s}}(\boldsymbol{X})
$$

其中, $E_{\mathrm{d}}\left(\boldsymbol{X}, \boldsymbol{X}_{\mathrm{L}}, \boldsymbol{Y}\right)$ 是约束项，表示 $\boldsymbol{Y}$ 在式(2)的线 性条件下, 重建的 HR $\boldsymbol{X}$ 能够变换到 $\boldsymbol{X}_{\mathrm{U}} ; E_{\mathrm{s}}(\boldsymbol{X})$ 是 $\boldsymbol{X}$ 的正则化先验项； $\lambda$ 是预定义参数，用以平 衡先验知识.

式(4)的 SR 重建模型线性约束项 $E_{\mathrm{d}}\left(\boldsymbol{X}, \boldsymbol{X}_{\mathrm{L}}, \boldsymbol{Y}\right)$, 在场景的可见光和红外之间的偏振态特征下具有 非线性不变特征, 可以通过学习获得. 为了对正则 化项 $E_{\mathrm{s}}(\boldsymbol{X})$ 建模，通常假设探测目标及场景包含稀 疏非一致性信息, 并且可以进行线性描述. 为此, 在 $\mathrm{CNN}$ 框架下, 通过设计空域约束获得 $C$, 并结 合正则化先验知识项完成偏振图像的 SR 重建.

\section{2 场景迁移 CNN 结构}

文献[21]表明，将 LR 近红外图像中提取的高 频组件作为 $\mathrm{CNN}$ 的多输人, 融合 $\mathrm{HR}$ 的可见光图 像，可以有效地提高红外图像的空间分辨率. 文献 [22-23]表明，全色图像空间像素值和光谱之间的 非线性映射比线性表示方法更有利于光谱重建. 因此，本文在 $\mathrm{CNN}$ 框架下，提出基于场景特征迁 移学习的空间调制型计算成像 SR 方法.

图 3 所示为空间调制型全偏振成像系统的 SR 网络结构. 该方法以可见光偏振为目标场景先验, 通过有效地学习非线性跨场景光谱表示, 在光谱 $\mathrm{CNN}$ 中利用多个卷积层学习可见光光谱与红外光 谱空间的非线性偏振特征映射的同时，对 HR 的可 见光偏振采用空间约束，以保证空间一致性.

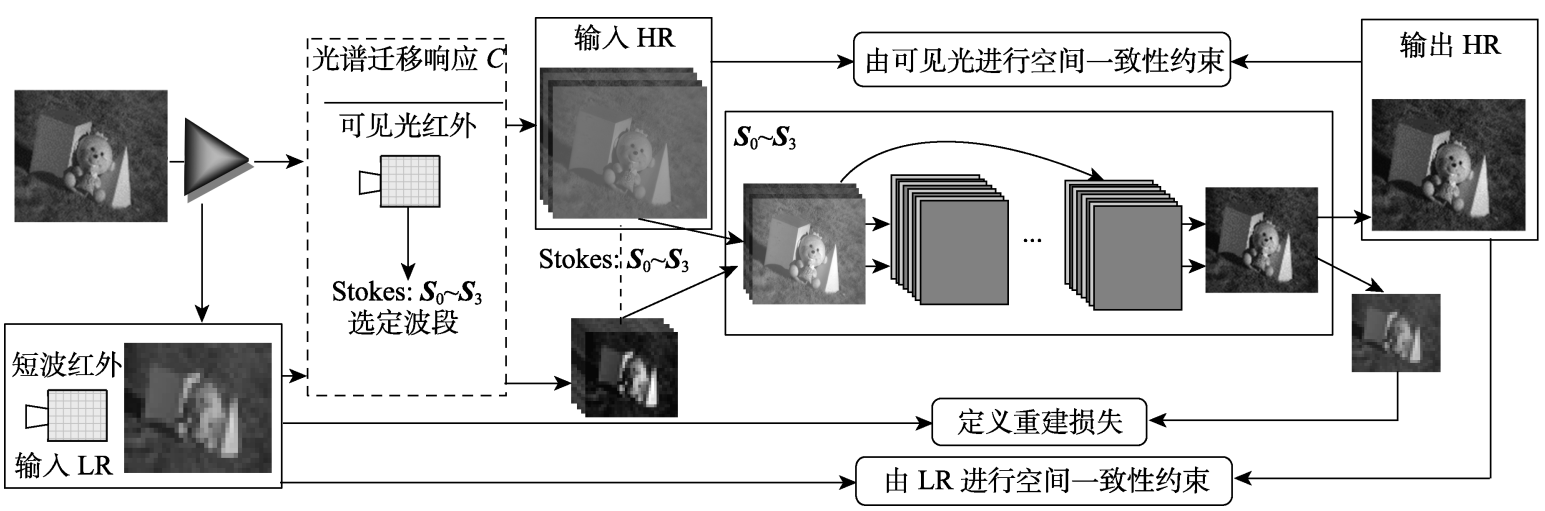

图 3 空间调制型全偏振成像系统的 SR 网络结构 
为获得可见光偏振图像和红外偏振图像之间 的光谱关系, 首先将 $L R$ 红外偏振图像 $\boldsymbol{X}_{\mathrm{L}}$ 通过光 谱迁移响应 $C$ 向下采样, 获得 LR 的可见光偏振图 像，即 $\boldsymbol{Y}_{\mathrm{L}}=C \boldsymbol{X}_{\mathrm{L}}$; 然后以获得的下采样可见光偏振 图像和 LR 红外偏振图像作为输人, 设计光谱 CNN 学习可见光偏振和相应的红外偏振光谱之间的非 线性映射. 同时，利用输人可见光图像指导空间分 辨率重建, 将输人的可见光图像与 $\mathrm{CNN}$ 各卷积层 的输出进行增强融合，建立空间信息重构模型. 光 谱 $\mathrm{CNN}$ 由 $L$ 层组成，则第 $l$ 层的输出激活函数表 示为

$$
F_{l}=g\left(K_{l} s\left(F_{l-1}, \mathbf{Y}\right)+b_{l}\right)
$$

其中, $g(x)=\max \{x, a x\} ; s\left(F_{l-1}, \boldsymbol{Y}\right)$ 表示将 $\boldsymbol{Y}$ 图像 与 $F_{l-1}$ 层进行输出叠加.

实际运算时令 $F_{0}=0$, 而参数 $a$ 的经验值设为 $a=0.05 . K_{l}$ 和 $b_{l}$ 分别表示第 $l$ 层的卷积核及偏差 项，根据经验设定 $L=5$, 为了学习光谱信息的内在 递归，卷积核的大小设为 $1 \times 1$.

将红外偏振 SR 网络的参数集记为 $W=\{K, b\}$, 设计最小化待重建的红外偏振 $\boldsymbol{X}_{\mathrm{L}}$ 和对应的真实图 像之间的均方误差(mean square error, MSE)为目标 函数, 实现参数训练

$$
L_{\mathrm{S}}(W)=\left\|f\left(\boldsymbol{Y}_{\mathrm{L}}, W\right)-\boldsymbol{X}_{\mathrm{L}}\right\|_{2}^{2}
$$

\section{3 分辨率一致性}

$\mathrm{HR}$ 可见光偏振和待重建的 HR 红外偏振图像 学习过程, 可以共享通过式(6)的模型学习获得的 参数. 在该参数条件下, $\mathrm{HR}$ 可见光偏振和待重建 的 HR 红外偏振图像的关系可以表示为

$$
\boldsymbol{X}=f(\boldsymbol{Y}, W)
$$

由式(2)(3)(7)可知, 待重建的 HR 红外偏振图 像线性映射后应该同时与 LR 红外偏振和 HR 的可 见光偏振保持一致，该一致性约束也用于学习模 型参数. 结合式(2)(3)(7), 在空域分辨率一致性约 束下，与式(6)对应的参数训练模型可以描述为

$$
L_{\mathrm{D}}(W)=\|\boldsymbol{Y}-C f(\boldsymbol{Y}, W)\|_{2}^{2}+\tau_{1}\left\|\boldsymbol{X}_{\mathrm{L}}-f(\boldsymbol{Y}, W) H\right\|_{2}^{2}
$$

可以看出，该模型将光谱非线性映射和空间分辨率 一致性纳人统一的框架来进行参数训练, 并通过优 化损失项恢复 HR 的红外偏振图像. 损失函数定义为

$$
L_{\mathrm{SD}}=L_{\mathrm{D}}(W)+\tau_{2} L_{\mathrm{S}}(W)+\eta_{1}\|W\|_{2}^{2}
$$

其中, 待重建的 HR 红外偏振图像的深层特征通过 深度先验学习代替人工设计先验项进行建模，深 层特征学习只在输人图像上进行, 无需对训练集 进行无监督学习。
式(9)的求解采用自适应矩估计方法 ${ }^{[24]}$. 其中, $\tau_{1}=50 ; \tau_{2}=50 ; \eta_{1}=10^{-4}$; 学习率初始值设置为 0.01 . 每层卷积层的权值 $V_{i j}$ 采用均匀分布 ${ }^{[25]}$ 来更 新, 即 $V_{i j} \sim U\left[-\frac{1}{\sqrt{n}}, \frac{1}{\sqrt{n}}\right]$. 其中, $U[-a, a]$ 为区间 $(-a, a)$ 的均匀分布; $n$ 为前一层的神经元大小.

\section{3 响应优化与重建联合学习}

\section{1 光谱迁移响应}

在第 2.1 节的场景特征迁移模型中, 将偏振光 谱从红外通道转换到可见光通道的场景偏振光谱 迁移响应函数 $C \in \mathbb{R}^{b \times B}$ 对重建质量有重要影响. 该 响应过程在图像复原中通常被建模为下采样并添 加噪声, 但是针对特定的高光谱全偏振成像系统, 这样的建模不具有适用性. 因此, 需要对光谱迁移 响应 $C$ 进行优化设计.

由式(3)可知, HR 可见光偏振图像与红外偏振 之间的线性关系描述为 $\boldsymbol{Y}=\boldsymbol{C} \boldsymbol{X}$. 本文借鉴文献[19] 的 RGB 与高光谱图像的光谱响应优化方法, 针对 高光谱全偏振调制成像系统，设计卷积层最优光 谱响应选择策略，以获得最佳光谱迁移响应，并将 光谱响应卷积优化层作为 SR 网络的前端输人, 与 全偏振 SR 重建联合学习。

在高光谱全偏振成像系统中, 第 $j$ 个光谱迁移 响应记为 $C_{j}$, 训练集中第 $t$ 个可见光偏振图像记 为 $\boldsymbol{X}_{t}$. 每个红外偏振对应光谱迁移的可见光偏振 图像与候选数据集中的所有光谱迁移响应函数合 成, 记为

$$
\boldsymbol{Y}_{j, t}=C_{j} \boldsymbol{X}_{t}
$$

获取不同场景的图像时，将不同光谱迁移响 应下的可见光偏振图像进行向量化，以构造输人 图像矩阵, 记为

$$
\boldsymbol{Y}_{t}=\left[\boldsymbol{Y}_{1, t}, \cdots, \boldsymbol{Y}_{j, t}, \cdots, \boldsymbol{Y}_{J, t}\right]
$$

显然, 最优光谱迁移响应函数 $C$ 应该使 $\boldsymbol{Y}_{t}$ 中 的合成可见光偏振图像 $C_{j} \boldsymbol{X}_{t}$ 最优. 如图 4 所示, 首 先将可见光偏振通道按照 Stokes 调制出 4 个分量 $\left(\boldsymbol{S}_{0}, \boldsymbol{S}_{1}, \boldsymbol{S}_{2}, \boldsymbol{S}_{3}\right)$, 然后使用共同的 $1 \times 1$ 卷积核 $V$ 进行 特征提取. 那么, 最优光谱迁移响应 $C$ 的网络输出 可以表示为

$$
\hat{\boldsymbol{Y}}_{t}=\left[V^{*} \boldsymbol{Y}_{t}\left(\boldsymbol{S}_{0}\right), V^{*} \boldsymbol{Y}_{t}\left(\boldsymbol{S}_{1}\right), V^{*} \boldsymbol{Y}_{t}\left(\boldsymbol{S}_{2}\right), V^{*} \boldsymbol{Y}_{t}\left(\boldsymbol{S}_{3}\right)\right]
$$
其中, $\boldsymbol{Y}_{t}\left(\boldsymbol{S}_{0}\right), \boldsymbol{Y}_{t}\left(\boldsymbol{S}_{1}\right), \boldsymbol{Y}_{t}\left(\boldsymbol{S}_{2}\right)$ 和 $\boldsymbol{Y}_{t}\left(\boldsymbol{S}_{3}\right)$ 分别表示 $\boldsymbol{Y}_{t}$ 的 空间调制型全偏振 Stokes 矢量通道，见式(1). 


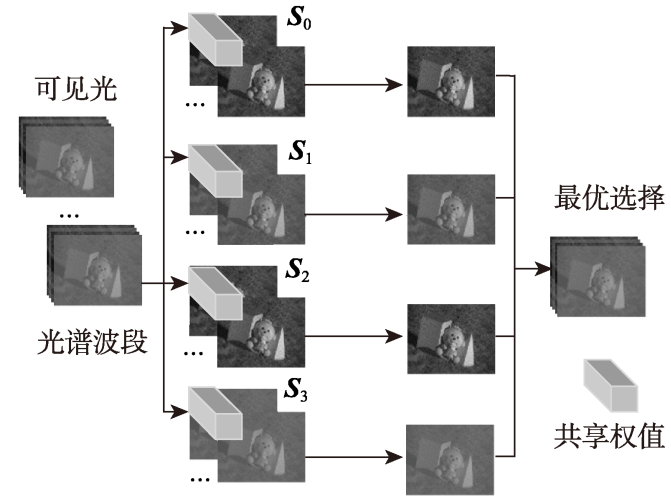

图 4 光谱迁移响应优化选择

由于仿真合成的可见光偏振图像应为正, $C$ 的选择权值应为非负, 因此在优化中引人稀疏性 约束. 而 $V$ 可以通过在非负稀疏约束下最小化在 选定的可见光偏振图像和对应真实图像之间的 MSE 确定，即

$$
\left\{\begin{array}{l}
L_{\mathrm{CS}}(V)=\frac{1}{T} \sum_{t=1}^{T}\left\|\hat{\boldsymbol{Y}}_{t}(V)-\mathbf{Y}_{t}\right\|_{2}^{2}+\eta_{2}\|V\|_{1} \\
\text { s.t. } V \geqslant 0
\end{array}\right.
$$

其中, $\hat{\boldsymbol{Y}}_{t}$ 为第 $t$ 个输出; $\boldsymbol{Y}_{t}$ 为第 $t$ 个真实图像; $T$ 为训练样本的数量. $V$ 值越大, 说明其对应的光谱 迁移响应对红外偏振的重建效果越好. 因此, $V$ 的 最大值对应的就是最优光谱迁移响应函数 $C$.

\subsection{SR 方法}

光谱迁移响应 $C$ 的优化层作为 SR 网络的前端 输人, 被附加到 SR 网络以优化 $C$, 该层与全偏振 SR 重建的参数联合学习. 为了在候选数据集中选 择最优 $C$, 通过最小化损失

$$
L=L_{\mathrm{CS}}(V)+\tau_{3} L_{\mathrm{SD}}(W)
$$

训练整个网络, 选择 $V$ 中值最大的作为最优光谱迁 移响应 $C$.

式(14)损失函数的求解同式(9), 采用自适应矩 估计方法 ${ }^{[24]}$. 实现时, 设置 $\tau_{3}=1, \eta_{2}=0.8, \mathrm{SR}$ 的学 习率设置为 0.001 . 为适合非负约束条件下 $C$ 的最 优选择, 卷积层的权值初始化为 $1 / J, J$ 为候选数 据集中的数量, 同式(11).

\section{4 实验结果与分析}

\section{1 实验环境及评价指标}

为了验证本文方法的有效性，采用多种场景 目标的多组偏振图像数据进行实验, 并与其他方 法进行比较.

实验硬件环境为训练用工控机环境， $17-7700+$
32 GB RAM CPU, NVIDIA Tesla T4 16GB GPU. 测 试环境为 HP Prodesk 480 G4 MT PC (i5-7500 (a) $3.40 \mathrm{GHz}$ CPU, $12 \mathrm{~GB}$ RAM); 网络训练工具采用 PyTorch, 运行于 Windows10 64 位操作系统.

成像系统为自行研制的非制冷像元耦合空间 调制型全偏振成像探测器，实验搭建环境如图 5 所 示, 偏振图像数据基本参数如第 1.1 节所述.

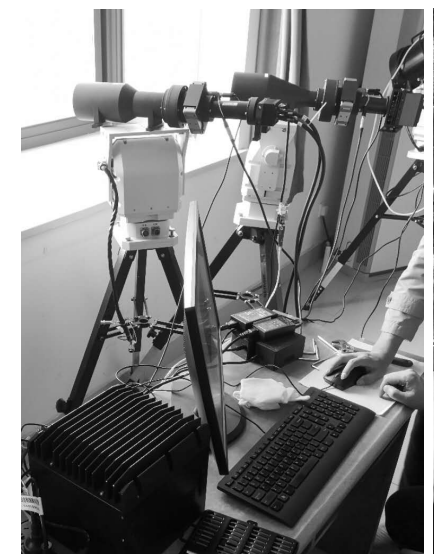

a. 实验平台

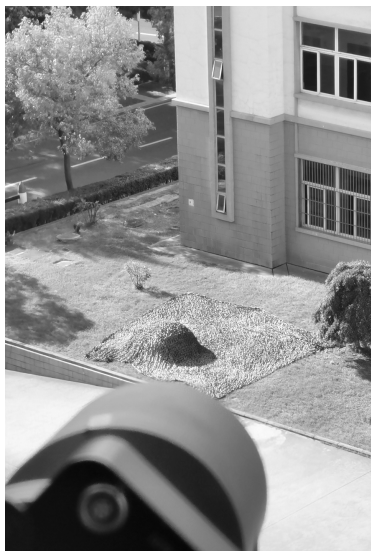

b. 实验外场景
图 5 实验平台及外场环境

实验包括 2 个方面:

(1) 采用实际成像系统进行同一场景的不同 分辨率数据采集, 以完成网络模型训练, 并通过与 其他方法进行仿真实验以验证本文方法的有效性.

(2) 将本文方法进行 SR 重建并与实际硬件采 集的真实图像进行比较, 根据误差调整系统参数, 为系统定标校正提供数据参考.

从主观视觉效果、客观量化评价指标以及偏振 参量解析结果 3 个方面进行评价. 主观评价从整体视 觉效果和局部纹理与轮廓结构进行; 客观评价指标 采用峰值信噪比(peak signal to noise ratio, PSNR)、光 谱角映射 (spectral angle mapper, SAM)、相关系数 (correlation coefficient, CC)、均方根误差(root mean squared error, RMSE)和结构相似性度(structural similarity index, SIM). 选择工程化常用的双三次插值 (Bicubic)、稀疏表示方法(Sparse) ${ }^{[26]}$ 和基于卷积神经 网络的超分辨率(super-resolution convolutional neural networks, SRCNN)算法 ${ }^{[27]}$ 进行对比.

\section{2 仿真实验}

图 6 所示为空间调制偏振系统采集的图像，解 析的偏振参量图只展示了偏振度参量. 成像系统 的像素级图像融合中, 系统的需求是将红外偏振 进行 3 倍 SR，以达到与可见光接近的空间分辨率， 而这也是比较普遍的中等规模 SR 倍率, 分别对原 始图和解调图进行实验. 


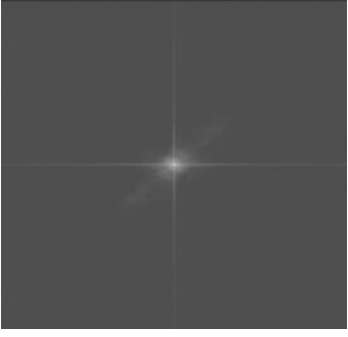

a. 频谱图

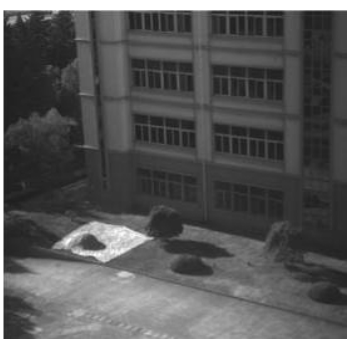

c. 解调图 $\boldsymbol{S}_{0}$

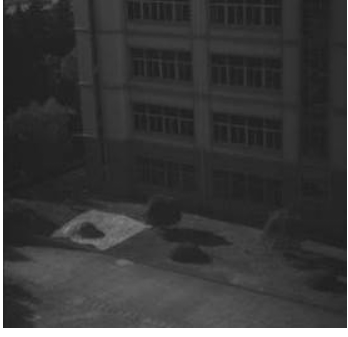

b. 原始图

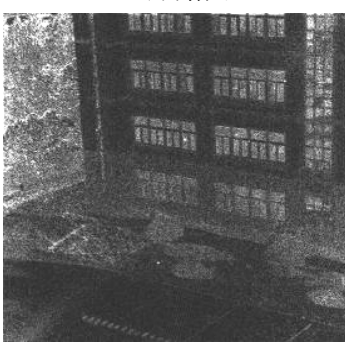

d. 偏振度参量
图 6 空间调制实验数据

图 7 所示为原始图的 3 倍 SR 实验结果, 仿真的 LR 图像由系统采集的最大分辨率经下采样和加噪声 获得, 为展示视觉效果, 对图像进行了裁剪操作. 可 以看出，图 7a 中伪装材料的表面明显被虚化; 而图 $7 \mathrm{c}$ 和图 $7 \mathrm{~d}$ 所示草地背景和建筑物墙体及马路边缘, 都取得了较好效果.

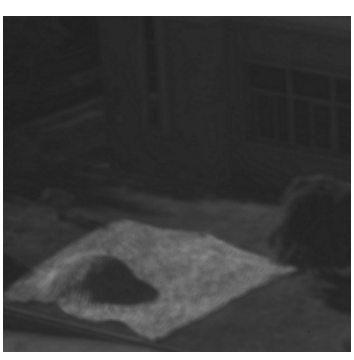

a. Bicubic

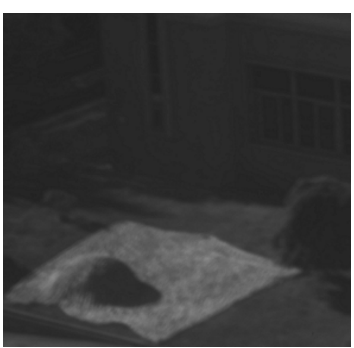

c. $\mathrm{SRCNN}^{[27]}$

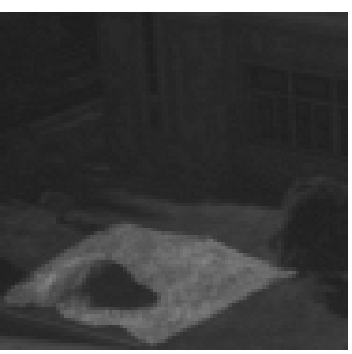

b. Sparse $^{[26]}$

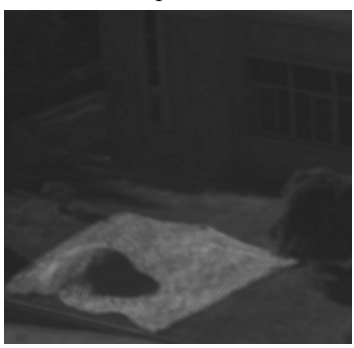

d. 本文
图 7 原始图实验结果

图 8 所示为解调图 $\left(\boldsymbol{S}_{0}\right)$ 的 3 倍 $\mathrm{SR}$ 实验结果, 其 中, 仿真的 LR 图像也是经下采样和加噪声获得, 为体现整体效果，此处显示缩放后的全图. 可以看 出, 图 $8 \mathrm{c}$ 和图 $8 \mathrm{~d}$ 都取得了优于图 $8 \mathrm{a}$ 和图 $8 \mathrm{~b}$ 的效 果; 但是通过建筑物窗户的边缘轮廓可以看出, 图 $8 \mathrm{c}$ 存在变形失真, 伪装网的纹理也趋于模糊.

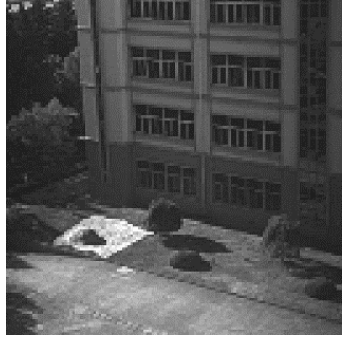

a. Bicubic

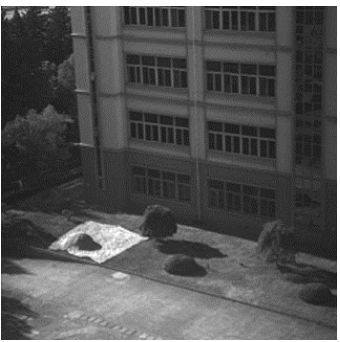

c. $\mathrm{SRCNN}^{[27]}$

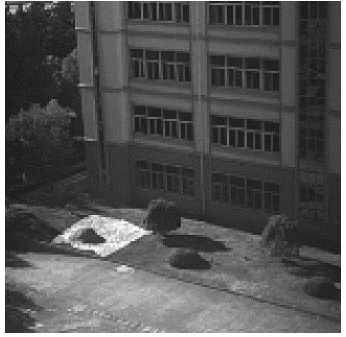

b. Sparse $\mathrm{S}^{[26]}$

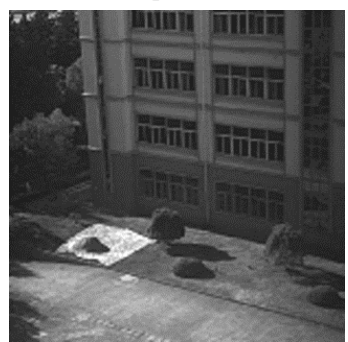

d. 本文
图 $8 \boldsymbol{S}_{0}$ 实验结果

为进一步评估 $\mathrm{SR}$ 重建结果, 采用客观评价指 标对图 7 和图 8 的图像进行评价, 结果如表 1 和表 2 所示. 可以看出，无论是表 1 还是表 2 ，本文方法 在大部分指标上都得了优于对比方法的效果; 同 时, 表 2 中进行 SR 重建优于在表 1 中重建的效果.

表 1 不同方法对原始图客观评价结果

\begin{tabular}{cccccc}
\hline 方法 & PSNR/dB & RMSE & SSIM & SAM & CC \\
\hline Bicubic & 26.34 & 1.10 & 0.78 & 0.051 & 0.93 \\
Sparse $^{[26]}$ & 25.45 & 1.13 & 0.79 & 0.053 & $\mathbf{0 . 9 6}$ \\
SRCNN $^{[27]}$ & 26.78 & 0.96 & $\mathbf{0 . 8 1}$ & $\mathbf{0 . 0 4 9}$ & 0.94 \\
本文 & $\mathbf{2 7 . 0 1}$ & $\mathbf{0 . 9 4}$ & $\mathbf{0 . 8 1}$ & 0.051 & $\mathbf{0 . 9 6}$ \\
\hline
\end{tabular}

注. 粗体表示最优指标数据.

表 2 不同方法对 $S_{0}$ 客观评价结果

\begin{tabular}{cccccc}
\hline 方法 & PSNR/dB & RMSE & SSIM & SAM & CC \\
\hline Bicubic & 26.87 & 1.03 & 0.81 & 0.050 & 0.95 \\
Sparse $^{[26]}$ & 26.35 & 1.01 & 0.83 & 0.051 & 0.96 \\
SRCNN $^{[27]}$ & 27.02 & 0.94 & 0.87 & 0.048 & $\mathbf{0 . 9 7}$ \\
本文 & $\mathbf{2 7 . 0 3}$ & $\mathbf{0 . 9 1}$ & $\mathbf{0 . 9 1}$ & $\mathbf{0 . 0 4 7}$ & $\mathbf{0 . 9 7}$ \\
\hline
\end{tabular}

注. 粗体表示最优指标数据.

\section{3 定标校正实验}

为适应不同视场目标探测和图像处理需要, 空间调制型全偏振计算成像系统的成像空间分辨 率可调. 对于分辨率为 $2048 \times 2048$ 的可见光偏振 和 $640 \times 512$ 的短波红外偏振，通过系统设置，分别 采集系统最大分辨率图像以及低空间分辨率图像; 然后对 LR(1 024×1 024 和 320×256)图像进行 SR, 
与实际采集的 HR 图像进行评价, 为偏振解调计算 成像系统的 SR 流程定型策略选择和系统定标校正 提供参考.

由第 4.2 节的实验结果可以看出，与在原始图 中进行重建相比, 在 $\boldsymbol{S}_{0}$ 中进行 SR 重建能够取得 优势效果. 因此, 对于该计算成像系统，选择在 解调后进行 SR 实验, 以重建后的全偏振解析结果 进行对比评价. 图 9 所示为系统采集的最大分辨 率图像与本文 SR 图像，并分别进行全偏振解析的 部分展示.

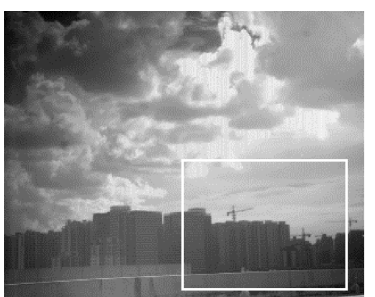

a. 全幅场景

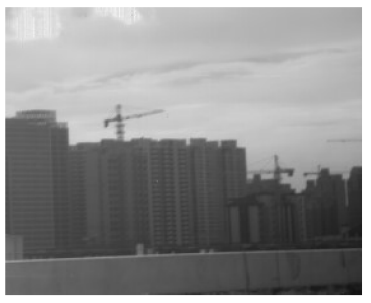

c. 系统HR

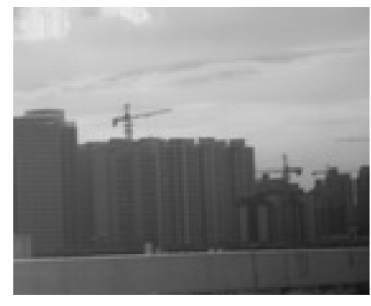

b. 系统 $L R$

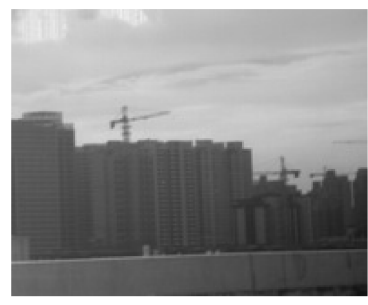

d. SR结果
图 9 系统 SR

系统采集的数据为 RAW 格式, 转换为 BMP 格 式全幅显示, 如图 9a 所示, 以便于观察实验结果 显示部分区域. 就整体视觉效果而言, 在 2 倍 SR 下的重建结果除了有少许的模糊外, 较系统采样 的 LR 图像有较大改善.

全偏振计算成像系统的最终结果是解析全偏 振参量. 为进一步验证本文方法, 将图 9 的实验进 行全偏振解析, 并以解析结果进行评价, 结果如图 10 所示, 图中只给出了偏振参量的偏振度图和 $\boldsymbol{U}$ 图解析结果. 可以看出, 它与图 9 所示 SR 结果几 乎一致, 在全偏振解析结果的视觉效果上取得了 逼近系统成像的结果.

对系统高分辨率(system HR, SHR)和 SR 重建 的全偏振解析结果采用无参考评价指标的清晰度、 信息熵以及对比度等进行评价, 结果如表 3 所示. 可以看出, 在对比度指标上, SR 结果普遍略低于系 统指标, 但是在清晰度和信息熵指标上, SR 结果基 本和系统原始结果保持一致.

\subsection{SR 融合实验}

如第 1.3 节所述，在实际成像探测应用中，通

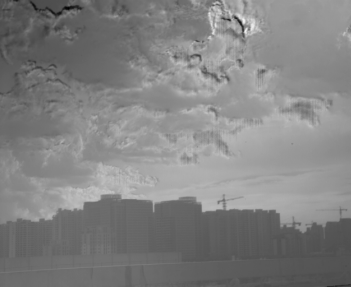

a. 系统偏振度图

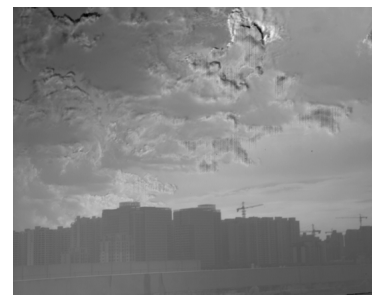

c. SR 偏振度图

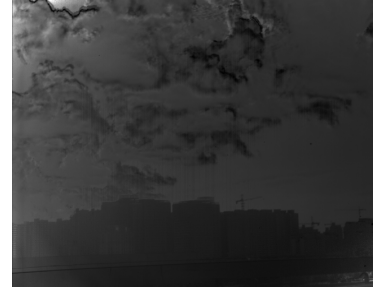

b. 系统 $\boldsymbol{U}$ 图

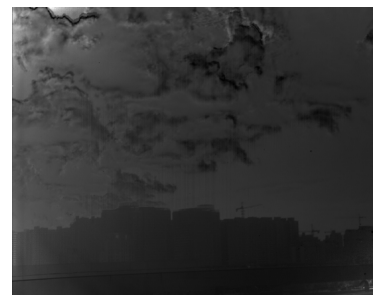

d. SRU 图
图 10 全偏振解析结果

表 3 全偏振解析无参考评价结果

\begin{tabular}{|c|c|c|c|c|c|c|}
\hline \multirow{2}{*}{ 评价指标 } & \multicolumn{2}{|c|}{ 偏振度 } & \multicolumn{2}{|c|}{$U$ 图 } & \multicolumn{2}{|c|}{$Q$ 图 } \\
\hline & SHR & SR & SHR & SR & SHR & SR \\
\hline 清晰度 & 0.37 & 0.39 & 0.24 & 0.24 & 0.21 & 0.21 \\
\hline 信息熵 & 6.25 & 6.25 & 6.47 & 6.72 & 6.62 & 6.61 \\
\hline 对比度 & 21.77 & 21.75 & 29.95 & 29.94 & 25.24 & 25.23 \\
\hline
\end{tabular}

注. 粗体表示最优指标数据.

常情况下为了综合利用多源图像特征以提高探测 和识别精度, 需要对图像进行融合处理. 本文的目 标之一是为空间调制型全偏振成像系统的探测目 标融合进行红外偏振图像 SR. 在飞行试验阶段, 首先对红外偏振图像进行 2 倍 SR 重建, 然后进行 伪彩色融合，通过最终的目标融合结果验证本文 方法的有效性. 红外偏振图像 SR 融合实验采用飞 行试验的对地探测数据进行, SR 融合结果如图 11 所示.

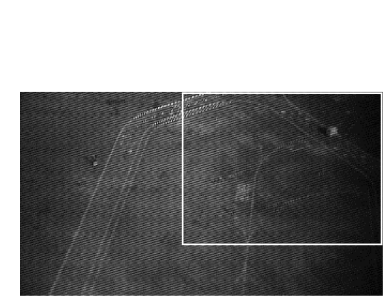

a. $\boldsymbol{Q}$ 图

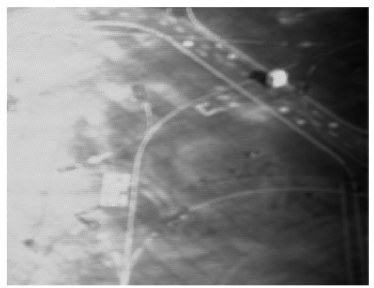

c. IHS融合

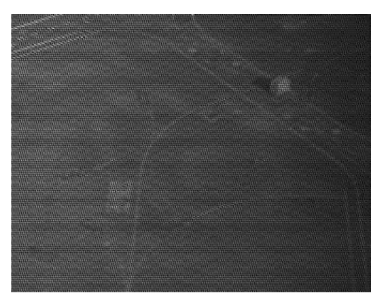

b. 自适应融合

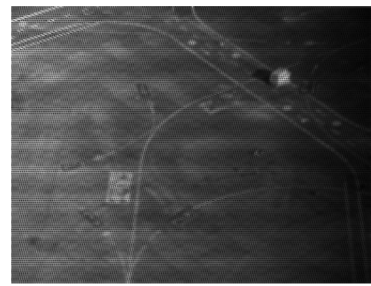

d. RGB融合
图 11 SR 融合结果 
图 11 中的目标为机场跑道及覆盖伪装网的车 辆. 由图 $11 \mathrm{a}$ 可以看出, 红外偏振信息得到有效保 持; 而从图 11b 图 11d 可以看出，在突显目标的同 时可以抑制杂散光干扰，起到“强光弱化，弱光强 化”的作用，反映在融合图像上，图像灰度分布更 加均匀, 伪装车辆、跑道分割线等目标更加突出.

由 SR 图像与系统 HR 图像的视觉效果、融合 结果和全偏振解析的客观评价指标数据可以看出, 采用场景迁移方法可以有效地改进计算成像系统 的图像空间分辨率限制; 同时根据实验结果可以 得出，在成像过程的空间调制与全偏振解析的中 间阶段进行 SR, 可以取得比较好的效果. 该结论 对实际偏振解调计算成像系统的 SR 流程定型策略 选择具有重要参考意义.

\section{5 结 语}

针对空间调制型计算成像降质过程及成像系 统的特点, 本文提出基于场景特征迁移学习的空 间调制型计算成像 SR 方法，可以提高系统的红外 偏振的空间分辨率. 通过改进场景特征迁移模型, 在空间分辨率一致性约束下选择最优光谱迁移响 应, 并作为 SR 网络的前端输人到 SR 网络, 与全偏 振 SR 重建的参数联合学习, 获得 HR 偏振图像. 通 过仿真实验和系统校正实验, 获得实际偏振解调计 算成像系统的 SR 定型策略选择依据, 且主观视觉 效果和客观评价指标都验证了本文方法的有效性.

虽然本文方法能够取得较好的分辨率提升效 果，但是在实时视频成像系统需求下，处理帧频无 法达到系统需求，只能以离线处理方式进行辅助 图像预处理. 今后，将进一步研究前端的调制计算 成像和实时在线处理算法，为实现高速率、 $\mathrm{HR}$ 的 空间调制型全偏振计算成像目标探测应用需求提 供支持. 本文方法对于提高偏振成像目标探测和 反演精度具有意义。

\section{参考文献(References):}

[1] Liu Zhen, Hong Jin, Gong Guanyuan, et al. Alignment error optimization of spatially modulated imaging polarimeter system[J]. Infrared and Laser Engineering, 2017, 46(1): 169-175(in Chinese)

(刘震，洪津，龚冠源，等. 空间调制型全偏振成像系统的角 度误差优化[J]. 红外与激光工程, 2017, 46(1): 169-175)

[2] Oka K, Kaneko T. Compact complete imaging polarimeter using birefringent wedge prisms[J]. Optics Express, 2003, 11(13):
1510-1519

[3] Ye Song, Yan Haofang, Sun Xiaobing, et al. Modified image demodulation algorithm for spatially modulated full-polarization imaging systems[J]. Acta Optica Sinica, 2019, 39(6): 74-80(in Chinese)

(叶松，严浩方，孙晓兵，等. 空间调制型全偏振成像系统的 图像解调算法修正[J]. 光学学报, 2019, 39(6): 74-80)

[4] Shao Xiaopeng, Liu Fei, Li Wei, et al. Latest progress in computational imaging technology and application[J]. Laser \& Optoelectronics Progress, 2020, 57(2): 3-47(in Chinese) (邵晓鹏，刘飞，李伟，等. 计算成像技术及应用最新进展 $[\mathrm{J}]$. 激光与光电子学进展, 2020, 57(2): 3-47)

[5] Xie Yisong, Li Zhengqiang, Hou Weizhen, et al. Retrieval of fine-mode aerosol optical depth based on remote sensing measurements of directional polarimetric camera onboard GF-5 satellite[J]. Aerospace Shanghai, 2019, 36(S2): 219-226(in Chinese) (谢一松, 李正强, 侯伟真, 等. 高分五号卫星多角度偏振成 像仪细粒子气溶胶光学厚度遥感反演[J]. 上海航天, 2019 , 36(S2): 219-226)

[6] Meng Xiangchao, Sun Weiwei, Ren Kai, et al. Spatial-spectral fusion of GF-5/GF-1 remote sensing images based on multiresolution analysis[J]. Journal of Remote Sensing, 2020, 24(4): 379-387(in Chinese)

(孟祥超，孙伟伟，任凯，等. 基于多分辨率分析的 GF-5 和 GF-1 遥感影像空-谱融合 [J]. 遥感学报, 2020, 24(4): 379-387)

[7] Xie Q, Zhou M H, Zhao Q, et al. Multispectral and hyperspectral image fusion by MS/HS fusion net[C] //Proceedings of the IEEE Conference on Computer Vision and Pattern Recognition. Los Alamitos: IEEE Computer Society Press, 2019: 1585-1594

[8] Wang W, Zeng W H, Huang Y, et al. Deep blind hyperspectral image fusion[C] //Proceedings of the IEEE International Conference on Computer Vision. Los Alamitos: IEEE Computer Society Press, 2019: 4150-4159

[9] Zhang L, Nie J T, Wei W, et al. Unsupervised adaptation learning for hyperspectral imagery super-resolution[C]//Proceedings of the IEEE Conference on Computer Vision and Pattern Recognition. Los Alamitos: IEEE Computer Society Press, 2020: 3070-3079

[10] Wang Z H, Chen J, Hoi S C H. Deep learning for image super-resolution: a survey[OL]. [2020-10-07]. https://arxiv.org/ abs/1902.06068v2

[11] Hu X C, Mu H Y, Zhang X Y, et al. Meta-SR: a magnification-arbitrary network for super-resolution[C] //Proceedings of the IEEE Conference on Computer Vision and Pattern Recognition. Los Alamitos: IEEE Computer Society Press, 2019: 1575-1584

[12] Soh J W, Cho S W, Cho N I. Meta-transfer learning for zero-shot super-resolution[OL]. [2020-10-07]. https://arxiv.org/ abs/2002.12213

[13] Zhang K, Zuo W M, Zhang L. Learning a single convolutional super-resolution network for multiple degradation[OL]. [2020-10-07]. https://arxiv.org/abs/1712.06116v2

[14] Bulat A, Yang J, Tzimiropoulos G. To learn image super resolution, use a GAN to learn how to do image degradation first[C] // Proceedings of European Conference on Computer Vision. Heidelberg: Springer, 2018: 187-202 
[15] Shocher A, Cohen N, Irani M. Zero shot super-resolution using deep internal learning[C] //Proceedings of the IEEE Conference on Computer Vision and Pattern Recognition. Los Alamitos: IEEE Computer Society Press, 2018: 3118-3126

[16] Sun Q R. Liu Y Y, Chua T, et al. Meta-transfer learning for few-shot learning[C] //Proceedings of the IEEE Conference on Computer Vision and Pattern Recognition. Los Alamitos: IEEE Computer Society Press, 2019: 403-412

[17] Arad B, Ben-Shahar O. Sparse recovery of hyperspectral signal from natural RGB images[C] //Proceedings of European Conference on Computer Vision. Heidelberg: Springer, 2016: 19-34

[18] Akhtar N, Mian A S. Hyperspectral recovery from RGB images using Gaussian processes[J]. IEEE Transactions on Pattern Analysis and Machine Intelligence, 2020, 42(1): 100-113

[19] Fu Y, Zhang T, Zheng Y Q, et al. Hyperspectral image super-resolution with optimized RGB guidance[C] //Proceedings of the IEEE Conference on Computer Vision and Pattern Recognition. Los Alamitos: IEEE Computer Society Press, 2019: 11661-11670

[20] Luo H T, Oka K, DeHoog E, et al. Compact and miniature snapshot imaging polarimeter[J]. Applied Optics, 2008, 47(24): 4413-4417

[21] Han T Y, Kim D H, Lee S H, et al. Infrared image su- per-resolution using auxiliary convolutional neural network and visible image under low-light conditions[J]. Journal of Visual Communication and Image Representation, 2018, 51(8): 191-200

[22] Jia Y, Zheng Y Q, Gu L, et al. From RGB to spectrum for natural scenes via manifold-based mapping[C] //Proceedings of the IEEE International Conference on Computer Vision. Los Alamitos: IEEE Computer Society Press, 2017: 4715-4723

[23] Choi I, Jeon D S, Nam G, et al. High-quality hyperspectral reconstruction using a spectral prior[J]. ACM Transactions on Graphics, 2017, 36(6): Article No.218

[24] Kingma D P, Ba J L. Adam: a method for stochastic optimization[OL]. [2020-10-07]. https://arxiv.org/abs/1412.6980v9

[25] Glorot X, Bengio Y. Understanding the difficulty of training deep feedforward neural networks $[\mathrm{J}]$. Journal of Machine Learning Research, 2010, 9: 249-256

[26] Yang J C, Wright J, Huang T S, et al. Image super-resolution via sparse representation[J]. IEEE Transactions on Image Processing, 2010, 19(11): 2861-2873

[27] Dong C, Loy C C, He K M, et al. Learning a deep convolutional network for image super-resolution[C] //Proceedings of European Conference on Computer Vision. Heidelberg: Springer, 2014: 184-199 